A N N A L E S Annales de Bretagne et des Pays de l'Ouest

Anjou. Maine. Poitou-Charente. Touraine

115-4 | 2008

Varia

\title{
La recherche à l'université de La Rochelle de 2000 à 2007
}

\section{(2) OpenEdition Journals}

Édition électronique

URL : http://journals.openedition.org/abpo/256

DOI : $10.4000 / a b p o .256$

ISBN : 978-2-7535-1512-3

ISSN : 2108-6443

Éditeur

Presses universitaires de Rennes

\section{Édition imprimée}

Date de publication : 31 décembre 2008

Pagination : $364-369$

ISBN : 978-2-7535-0808-8

ISSN : 0399-0826

\section{Référence électronique}

"La recherche à l'université de La Rochelle de 2000 à 2007 », Annales de Bretagne et des Pays de l'Ouest [En ligne], 115-4 | 2008, mis en ligne le 31 décembre 2009, consulté le 14 septembre 2020 URL : http://journals.openedition.org/abpo/256

Ce document a été généré automatiquement le 14 septembre 2020

(c) Presses universitaires de Rennes 


\section{La recherche à l'université de La Rochelle de 2000 à 2007}

1 Liste des thèses et mémoires soutenus et déposés à l'université de La Rochelle.

\section{Mémoires}

2 JOSEFIAK, Caroline, Les églises fortifiées des côtes d'Aunis, un guet de la mer autour de La Rochelle, dir. N. FAUCHERRE.

3 LivenAIS, Juliette, La mise en place de la maison de la culture de La Rochelle au théâtre Municipal 1966-1973 : enjeux et évolutions, dir. G. MARTINIÈRE.

4 Michaud, Laëtitia, L'architecture de la fonderie de canons de bronze de Rochefort (1668-1720), dir. M. ACERRA.

5 MONET, Jean-Sébastien, La femme face au crime en Charente inférieure dans le dernier tiers du XIX siècle, dir. L. VIDAL.

6 MONROSTY, Benoit, Le ravitaillement à La Rochelle pendant la seconde guerre mondiale, dir. $\mathrm{L}$. VIDAL.

7 MORIN, Élise, Les ouvriers de l'Arsenal de Rochefort (1783-1789), dir. M. ACERRA.

8 Petrowiste, Tony, Jean Fe de Segeville : un noble de province au XVIIe et au XVIII siècle, dir. M. ACERRA.

9 PoITIER, Sylvain, La politique sociale de Francia, dictateur du Paraguay (1814-1840) Un despotisme éclairé ?, dir. L. VIDAL.

10 POURCIN, Céline, Histoire topographique de Saint Jean d'Angely, dir. N. FAUCHERRE.

11 Roy, Nicolas, La société de loisirs à La Rochelle au XVIII siècle, dir. M. ACERRA

2001

BAUDIN, Clara, La scolarisation des filles à Saint Xandre 1876-1881, dir. L. VIDAL. 
BAUDOUIN, Valérie, Politique et sociabilités dans l'arrondissement de Parthenay (Deux Sèvres) : 1889-1914, dir. M. AUGERON.

BENDADA, Ségolène, La solidarité internationale rochelaise vue à travers le prisme de l'engagement de trois associations locales (1961-1995), dir. L. VIDAL.

BONNEAU, Christèle, Occupation du sol au sud de la Charente-Maritime dans l'antiquité, dir. L. TRANOY.

BONNEAU, Loïc, L'asile départemental d'aliénés de Lafond et la folie en Charente-inférieure, de 1829-1893, dir. C. ILLOUZ.

BORTHAIRE, Cédric, Les gens de mer de l'île royale, vie et mœurs :1713-1758, dir. M. ACERRA.

Bouchon, Frédéric, De Halle à Halle Neustadt, de la ville hanséatique à la ville nouvelle. Urbanisme et société en Allemagne de l'Est, dir. L. VIDAL.

BRUSTIS, Nadège, Les loisirs à La Rochelle XIIIe-XVe siècles, dir. M. TRANCHANT.

EyBrALY, Guylaine, La pêche à La Rochelle de 1958 à 1994 : étude d'un port en crise, dir. M. AUGERON.

GERBIER, Christophe, La vie religieuse de la population rochelaise de 1804 à 1870, dir. M. AUGERON.

GoRIN, Christian, La Rochelle-Palice. Escale transatlantique: perspectives et réalités (1860-1960), dir. M. Augeron.

GREGOR, Thierry, La mise en œuvre de la pierre à Saintes à l'époque antique, dir. L. TRANOY.

HERNANDEZ, Céline, La présence économique française dans les territoires palestiniens, 1994-2000, dir. M. Augeron.

JACQUET, Aurélie, Le renouveau de l'idée coloniale à Rochefort, 1878-1904. Le cas de la loge " l'Accord Parfait ", dir. L. VIDAL.

Jaumouillie, Anne-Laure, Le fabuleux destin de Claude Petitjean, communard, bagnard et colon en Nouvelle-Calédonie (Paris1845 - Hienghène 1914), dir. C. ILlouz.

27 MAGRE, Julie, Images de l'île de Ténériffe de la France révolutionnaire à la France de l'Empire (1789-1813), dir. M. AugERON.

28 MARZE, Anne-Sophie, Eléments pour une historiographie de l'immigration russe en France, de 1917 à nos jours, dir. L. VIDAL.

9 MoINE, Céline, Étude d'un lot de céramiques provenant du site de Barzan (Charente-Maritime), dir. L. TRANOY.

o Morillon-Briere, Sébastien, Bernard Charbonneau et le totalitarisme : 1910-1950, dir. L. VIDAL.

1 NAUD, Mathieu, La société de statistiques puis historique des Deux-Sèvres et le patrimoine du marais Poitevin (1836-1986), dir. G. MARTINIÈRE.

NocEDAL, Doris, Les métamorphoses en Grèce, dir. A. Coulié.

Pouponnot, Guillaume, L'aumônerie Saint-Berthélemy de La Rochelle au XV siècle, dir. M. TRANCHANT.

4 REINERT, Carine, Les La Trémoelle et leurs territoires en Aunis au XVe siècle, dir. M. TRANCHANT. 
RoBin, Bruno, Portraits de femmes : les marginales chez les Aztèques de la période précolombienne à la conquête, dir. M. AUGERON.

RONSSERY, Céline, Étude de la correspondance par Le Sieur Jacques François Arthur 1er médecin du roi à Cayenne (1736-1771), dir. M. AUGERON.

\section{THEBAUT, Evelyne, Topographie historique de l'île de Maillezais, dir. N. FAUCHERRE.}

ThEolissat, Marie-Noëlle, L'échevinage rochelaise au xve siècle, dir. M. TRANCHANT.

TISSERAND, Stéphane, L'Afrique et les Africains vus par les Français : La Sénégambie à travers la littérature de voyages des XVII et XVIII siècles, dir. C. ILLOUZ.

ZunzunEGUI, Caroline, La présence française à Ilhéus (Bahia-Brésil). Le cas de la famille Lavigne, dir. L. VIDAL.

BARRES, Jean, La bourgeoisie protestante rochelaise dans la première moitié du XVIII siècle, dir. M. AUGERON.

\section{2}

BonNIN, Guillaume, La Chine et les chinois vu par un français au XIXe siècle : voyage d'un rochelais " rochelais dévoué ", le Baron de Chasiron lors de la mission française en extrèmeorient (1857-1861), dir. C. ILlouz.

CANTELejo, Cyril, Les conséquences de l'indépendance sur la vie quotidienne des Conakrycas de 1858-1977, dir. L. VIDAL.

CHARBONNEAU, Jean-Paul, Le système hydraulique de la forteresse de Salses, dir. N. FAUCHERRE.

Chassagnac-Mazza, Françoise, Les sans-abri à La Rochelle de nos jours, dir. L. VIDAL.

CHIKLI, Christophe, Apogée politique et sociale d'une élite anglophone: les maires de Sherbrooke (Quebec) 1852-1900, dir. M. AugERON.

CLAVERY, Aude, L'île d'Aix de Louis XV au $1^{\text {er }}$ Empire : Populations et activités en temps de guerre, dir. M. ACERRA.

DeBelly, Sabrina, Auguste Hardisson ou la réussite d'un migrant français à Ténériffe (1842-1883), dir. M. AUGERON.

DEMUYNCK, Thibault, Royan, station balnéaire (1870-1914), dir. L. VIDAL.

FLUTTO, Laurent, Projets de ville, projets de sociétés, contribution à l'histoire urbaine à travers l'exemple de la politique sportive (1844-2001), dir. M AUGERON.

GAILLARD, Nathalie, Le Haras national de Saintes (1844-1999), dir. M. AUGERON.

GAREAUD, Celine, Étude d'un village charentais de Saint-Agnant les marais entre 1850-1890, dir. M. AUGERON.

GRENE, Cedric, Les hôtels particuliers de Rochefort sous l'Ancien Empire : l'apparition d'un modèle et son contexte architectural, dir. M. ACERRA.

54 GRIzON, Émilie, La criminalité en Charente-Maritime-Inférieure sous le Second Empire, dir. M. AUGERON.

GUILloteAu, Marilyne, Le site de Broue, dir. N. FAUCHERRE.

HeYLBRoECK, Julien, Les différences de relation à la mort entre Occident et Japon à travers les multiples interprétations du suicide de Mishima Yukio, dir. C. Illouz. 
HILLAIRET, Aurore, Les bourgeois rochelais peints par Louise Rang-Babut et André Brossard, dir. M. AugERON.

LARBAT, Mickael, La garde nationale de La Rochelle 1831-1871, dir. M. AUGERON.

MAGNIN, Charlotte, Les religieuses à La Rochelle et la reconstruction congréganiste (1801-1881), dir. M. AugERON.

MARCET, Sylvia, "Les Déjhouqués " : la survivance d'un groupe et de son folflore, dir. L. VIDAL.

MoIZAN, Emmanuel, La rue et ses aménagements dans l'agglomération de l'Aquitaine antique, dir. L. TRANOY.

NORIGEON, Franck, La bibliothèque municipale de La Rochelle (1833-1939), dir. M. AUGERON.

PELLETIER, Arnaud, État des ouvrages que le Roy ordonne estre faits à la citadelle d'Oléron (1685-1693), dir. M. ACERRA.

64 Penaud, Christelle, La criminalité dans l'arrondissement de Jonzac (1860-1900), dir. M. AUGERON.

5 PERON, Francis, Espaces naturels et activités humaines en île d'Oléron du XIe au XVI siècle, dir. M. TRANCHANT.

6 Poitoizeau, Sébastien, Lieux, expressions et enjeux de la mémoire sherbrookoise, dir. M. AUGERON.

67 POTIER, Samuel, Enjeux, valeur et histoire mouvementée du Traité sue quelques points de la religion des chinois du Père Niccolo Longobardi, dir. M. AUGERON.

68 SERRURIER, Herve, Mémoire du Général Chasseloup sur les places du royaume d'Italie, dir. L. VIDAL.

SOUIAH, Karim Malik, L'appropriation culturelle des monuments de la ville de La Rochelle par la municipalité, dir. M. AUGERON.

o VAUNAT, Virginie, Étienne Laurent Dechezeaux, négociant à La Flotte : Étude d'un négoce pratiqué hors des grands ports français du XVIII siècle, dir. M. AUGERON.

\section{3}

71 BERIET, Grégory, Le bagne de Rochefort à l'épreuve du débat pénitentiaire (1815-1852) : institution de répression et économie coercitive, dir. L. VIDAL.

72 BERNHARD, Nathalie, Enquête sur les privilèges de Libourne (1486-1487). Edition critique et présentation historique, dir. M. BOCHACA.

73 BRIGNON, Julien, Le refuge huguenot en Afrique du sud : historiographies françaises et sudafricaines sur la question. Un bilan historiographique, dir. M. AUGERON.

74 CARIOU, Pierrick, La ligue communiste révolutionnaire (LCR) en Charente-Maritime dans les années 70, dir. L. VIDAL.

75 CouvidAT, Janique, Réalité, impact et pouvoir du nouveau corps de ville rochelais (1695-1698), dir. M. AugERON.

76 CUDENNEC, Guillaume, Morphogenèse de Tonnay-Boutonne. Formes et monuments d'un village médiéval (Xe-XIXe siècles), dir. M. AUGERON. 


\section{4}

DELMON, Romain, Géostratégie et diplomatie française sur les littoraux antillais et nordaméricains : étude de la division de l'atlantique (1885-1888), dir. M. AUGERON.

El Mamoune, Hamid, La Rochelle sous l'occupation allemande (1940-1941), dir. L. VIDAL.

GANDOUET, Denis, La vente des biens nationaux de première et de seconde origine dans le district de Rochefort, dir. M. AugERON.

GAULMIER, Catherine, Librairies à Rochefort et à La Rochelle de 1810 à 1870, dir. M. AUGERON.

LICOP, Audrey, Les rapports entre l'histoire et la justice au procès de Paul Touvier, dir L. VIDAL.

MoRIN, Sandrine, Sarah Golberg, de l'orchestre rouge à Auschwitz-Birkenau : une femme en résistance, dir. M. AUGERON.

PERONNEAU, Magali, La « réconciliation » de l'université de la ville : le cas de la création de la Faculté de Lettres, Langues, Arts et Sciences Humaines (FLASH) de l'université de La Rochelle, dir. L. VIDAL.

PICQ, Mathias, René Château de la gauche à Pétain, un itinéraire politique dans les années 30-40, dir. L. VIDAL.

PLEZ, J.-Philippe, Étude autour d'une cause de béatification : le cas de Marie Eustelle Harpain (1814-1842), dir. C. Illouz.

POUSSARD, Aurélia, Le développement économique de la compagnie des bateaux à vapeur dite " union oléronnaise et rochelaise " à travers l'administration des deux gérants successifs : Jules Fournier et Ernest Raoulx (1892-1940), dir. M. AUGERON.

SABAUT, Magali, Le jumelage à La Rochelle, dir. M. Augeron.

VAUDON, Anthony, Les associations culturelles à La Rochelle (1950-1983), dir. M. AUgERON.

ANQUETIL, Frédéric, Le fort de la rade. Histoire et évolution défensive (1692-1814), dir. N. FAUCHERRE.

ARnOuX, David, La politique municipale à La Rochelle de 1848 à 1900, dir. M. AUGERON.

APCHER, Laure, Lumière sur le palace : le film sans fin d'une salle de cinéma en milieu rural (Sugères, 1909-2004), dir. L. VIDAL.

BERNARD, Marjorie, Mémoire de témoins et occupation à La Rochelle (juin 1940-mai 1945), dir. C. Illouz.

CAILlERE, Élise, Étude d'un projet de diffusion musicale et de développement culturel urbain : Le Festival des francofolies de La Rochelle, 1958-2004, dir. L. VIDAL.

Coppin, Laurie, Étude prosopographique des gens de mer dans la quartier maritime de La Rochelle au XIXe siècle (1814-1891), dir. M. AUGERON.

DaViauX, Anne-Lise, La place de l'hôtellerie de tourisme dans l'évolution de la station balnéaire de La Rochelle (1850-1965), dir. L. VIDAL.

DINAND, Laëtitia, La morphogenèse de Jonzac des origines au XIXe siècle : compréhension Historique du patrimoine urbain d'une petite ville saintongeaise, dir. M. AUGERON.

DUGAST, Amélie, La communauté protestante rochelaise sous la révolution (1787-1802), dir. M. AUGERON. 

dir. M. TRANCHANT.

\section{2}

113 EYBRALY, Guylaine, La sensibilité des Rochelais face à la mort, du décret du 23 prairial An 12 à la veille de la Grande Guerre, dir. G. MARTINIERE.

114 FRICHE, Claude, Les possessions monastiques sur le littoral de l'Aunis et de la Saintonge, de la fin du XIIIe siècle jusqu'au début du XVI ${ }^{e}$ siècle, dir. M. BOCHACA, co-dir. M. TRANCHANT.

115 GAILlARD, Jacques, L'exploitation traditionnelle de la pierre des carrières du Val de Charente depuis l'Antiquité jusqu'au milieu du $\mathrm{XX}^{e}$ siècle : Essai de modélisation de la période 1840-1960 à partir des carrières de Thénac en Charente-Maritime, dir. N. FAUCHERRE. 
116 Issoufali, Abdoul Karim, Les sociétés coloniales dans la formation économique et sociale des Comores : 1860-1960, dir. G. MARTINIERE.

117 Jaumouillie, Anne-Laure, De la rencontre des cultures Kanak et coloniale dans la genèse des systèmes relationnels calédoniens au $\mathrm{XX}^{e}$ siècle, dir. G. MARTINIERE.

118 PlouvIEZ, David, Les approvisionnements de la marine de guerre française au XVIII siècle : Les relations entre l'État et la Compagnie de fourniture de pois Péan de Saint-Gilles (1761-1770), dir. M. ACCERA.

119 Pourcin, Céline, L'aménagement des berges de la rivière Boutonne avant la Révolution Française : économie, peuplement et urbanisation d'une voie de communication naturelle, dir. N. FAUCHERRE. RoBIN, Bruno, Les Indiens et la mer (1300-1600), dir. M. ACERRA, co-dir. M. AUGERON.

121 RONSSERAY, Céline, Le personnel administratif en poste en Guyane Française (1720-1820), dir. G. MARTINIERE.

122 Theolissat, Marie-Noëlle, Le notariat rochelais à la fin du Moyen-Age à travers l'exemple du Notaire Boutin, dir. M. BOCHACA, co-dir. M. TRANCHANT.

\section{3}

BADARO, Jane, Communication touristique et lieu de mémoire. Essai sur la représentation de deux villes atlantiques au XXe siècle: La Rochelle (FRANCE) et ILHEUS (BAHIA), dir. G. MARTINIERE.

BouILLAUD, Gaëllle, Te Tapa Tongarewa, Musée national de Nouvelle-Zélande, de l'idée à nos jours : Histoire, société, culture et muséologie, dir. G. MARTINIERE.

125 Cantalejo, Cyril, Les origines et les débuts de la mise en place de la politique française de coopération en Afrique : les cas du Dahomey, du Togo, de la Côte d'Ivoire et du Sénégal (1958-1973), dir. G. MARTINIERE.

126 Chassagnac-Mazza, Françoise, L'hégémonie américaine et l'Union Européenne (1991-2002), dir. G. MARTINIERE.

127 Magnin, Charlotte, Les missionnaires vendéens en Outre-Mer sous l'épiscopat de Monseigneur Catteau (1877-1915), dir. G. MARTINIERE.

128 MARCET, Sylvia, Folklore, identités et sociabilités : étude comparée de 3 groupes folkloriques (Québec, France, Brésil) (1970-c.2003), dir. G. MARTINIERE, co-dir. M. VIDAL.

Ould Mohamed, Sidi Mohamed, La pêche en Mauritanie depuis l'Indépendance (1960-2000), dir. G. MARTINIERE.

PENAUD, Christelle, La justice et les gens de mer au XIX siècle en Charente-Inférieure (1852-1900), dir. G. MARTINIERE.

131 SAZERAT, Élise, La vision occidentale de l'Autre : les collectionneurs d'objets ethnographiques du Museum d'Histoire Naturelle de La Rochelle, dir. G. MARTINIERE.

SOUIAH, Karim Malik, La mise en scène du patrimoine : le cas de la Charente-Maritime (des années soixante au début des années 1990). L'évolution des politiques de mise en valeur, dir. G. MARTINIERE. 
2004

\section{Master 2} dir. M. BOCHACA.

BERIET, Grégory, L'école de médecine navale de Rochefort au XIXe siècle, dir. G. MARTINIERE.

BRIAND-MELISSON, Céline, Les officiers du roi en Nouvelle-France. Pouvoirs et sociabilités des fonctionnaires coloniaux (1663-1760), dir. G. MARTINIERE.

DANIEL-MARTINIERE, Irène, Les Américains à La Rochelle (1950-1964), dir. D. Poton.

FOURNIER, Caroline, La criminalité à Rochefort sur Mer de 1811 à 1914, dir. G. MARTINIERE.

GalouZeaude V illepin, Aurélia, Le regard porté par les Français sur la Malaisie : " les écrivains-planteurs de caoutchouc " français, de 1905, arrivée de Henri Fauconnier à 1957, indépendance du pays, dir. G. MARTINIERE.

GuILLEMIN, David, Mécanismes de représentation comparés du Japon et de la Chine en France du début du XVIIIe à Emile Guimet (1729-1878), dir. G. MARTINIERE.

HiLlaIRET, Aurore, Les élites culturelles rochelaises au XIXe siècle, dir. G. MARTINIERE.

LEMBANG, Hendricus, L'Indonésie dans le contexte du régionalisme. Coopération de l'ASEAN en face d'un nouvel ordre mondial, dir. D. РотоN.

PERISSE, Sébastien, La reconstruction d'une région littorale : la Saintonge méridionale à la fin du Moyen-Age (vers 1440-vers 1530), dir. M. BосHACA.

PLEZ, Jean-Philippe, Saints et sainteté en France de l'Ouest et du Canada francophone (xIxe-Xxe siècles), dir. D. POTON.

Pouponnot, Guillaume, L'activité économique dans la basse vallée de la Charente au MoyenAge (xie- $\mathrm{Xv}^{e}$ siècle), dir. M. BOCHACA.

RoY, Jean-Philippe, L'émigration française vers les États-Unis: Tendances d'hier et d'aujourd'hui, dir. D. РотоN.

THOMAs, Sophie, Bordeaux et sa banlieue (1451-1469). De la "ville traître " à la bonne ville,

\section{5}

ANDERSON, Melanie, La question d'alliances et la lutte pour l'opinion de la communauté internationale vues à travers la presse argentine et britannique pendant la guerre des îles Malouines ( $1^{\text {er }}$ avril au 14 juin 1982), dir. G. MARTINIERE.

147 ANGSAR, Angela, La politique de peuplement en Nouvelle-Calédonie : du bagne à la colonie vers une identité territoriale, dir. G. MARTINIERE.

Deniau, Vanessa, La perception de l'autre dans la littérature et l'iconographie pendant les grandes découvertes en Amérique (XVIe-XVII siècle), dir.G.MARTINIERE.

149 GIORCELLI, Sabrina, Évolution des relations culturelles franco-italiennes au travers des Biennales de Venise d'art figuratif et d'art cinématographique (1946-1970), dir. G. MARTINIERE.

150 NERROU, Kristell, Rochefort, porte des Antilles. Les relations entre l'arsenal et les colonies (1777-1780), dir. M. ACERRA. 
151 OULD N'TAHAH, Mohamed Salem, Les relations Franco-Mauritaniennes à partir de la seconde moitié $d u X^{e}$ siècle, dir. G. MARTINIERE.

PERAUD, Emmanuel, La délinquance dans l'arrondissement de La Rochelle de 1951 à 1955, dir. D. РОTON.

SCHREIBER, Loic, L'action culturelle de la France en République Fédérale d'Allemagne (1945-1968), dir. G. MARTINIERE.

SCHUGt, Alexander, L'administration de Jimmy Carter, la France et l'OTAN (1976-1981), dir. F. Bozo.

\section{6}

BACHE-GABRIELSEN, Pierre, Approche prosopographique des conseillers municipaux de La Rochelle élus à l'aube du Second Empire, dir. D. POTON.

Boutin, Jean-Charles, Un pouvoir municipal en rébellion : le corps de ville de La Rochelle, 1622-1625, dir. D. РотоN.

157 BRAUD, Padrig, Bande dessinée et histoire, La représentation de l'espace atlantique aux XVIeXVIII ${ }^{e}$ siècles dans les albums de Bande dessinée d'expression française (1943-2005), dir. D. POTON.

CAILLAUD, Benjamin, Fernand Braun (1852-1948), photographe républicain à Royan, dir. G. MARTINIERE.

GASPARD, Lenique, Les slogans et les graffitis politiques à Port-au-Prince (Haïti) : 1986-2004, dir. G. MARTINIERE.

160 MalfoY, Cécile, Sortir du royaume : l'exemple des protestants charentais et poitevins (1660-1760), dir. D. РотоN.

161 MoRillon, Sébastien, Les historiens français et les États-Unis dans la seconde moitié du XX siècle : Regards, expériences, rencontres, échanges... (contribution à l'histoire des échanges intellectuels transatlantiques), dir. L. VIDAL.

PORCHER, Kévin, La tonnellerie bordelaise après la guerre de Cent ans, dir. M. BOCHACA. Véracruzana, dir. D. РотоN.

164 UtAmA, Sandjaja, Comment les navigateurs voient Sumatra au XVI e siècle à travers les travaux de Parmentier, dir. G. MARTINIERE.

\section{7}

BACHE-GABRIELSEN, Pierre, Approche prosopographique des conseillers municipaux de La Rochelle élus à l'aube du Second Empire, dir. D. РотоN.

Boutin, Jean-Charles, Un pouvoir municipal en rébellion : le corps de ville de La Rochelle, 1622-1625, dir. D. РотоN.

BRAUD, Padrig, Bande dessinée et histoire, La représentation de l'espace atlantique aux XVIeXVIII ${ }^{e}$ siècles dans les albums de Bande dessinée d'expression française (1943-2005), dir. D. POTON. 
CAILlAuD, Benjamin, Fernand Braun (1852-1948), photographe républicain à Royan, dir. G. MARTINIERE.

GASPARD, Lenique, Les slogans et les graffitis politiques à Port-au-Prince (Haïti) : 1986-2004, dir. G. MARTINIERE.

Malfoy, Cécile, Sortir du royaume: l'exemple des protestants charentais et poitevins (1660-1760), dir. D. РотоN.

171 MoRillon, Sébastien, Les historiens français et les États-Unis dans la seconde moitié du XX siècle : Regards, expériences, rencontres, échanges... (contribution à l'histoire des échanges intellectuels transatlantiques), dir. L. VIDAL.

PORCHER, Kévin, La tonnellerie bordelaise après la guerre de Cent ans, dir. M. BOCHACA.

173 TEXIER-POULAIN, Laëtitia, La criminalité au Mexique au $\mathrm{XX}^{e}$ siècle : l'exemple de la Huasteca Véracruzana, dir. D. РотоN.

174 UtAmA, Sandjaja, Comment les navigateurs voient Sumatra au XVI e siècle à travers les travaux de Parmentier, dir. G. MARTINIERE.

\section{Thèses}

\section{7}

GAILLARD, Jacques, L'exploitation antique de la pierre de taille dans le bassin de la Charente, dir. M. BOCHACA.

JAUMOUILLÉ, Anne-Laure, Entre sagaïes et médailles : processus colonial de reconnaissance des chefs kanak en Nouvelle-Calédonie (1878-1946), dir. G. Martinière et C. ILLouz.

177 RONSSERAY, Céline, Administrer Cayenne : sociabilités, fidélités et pouvoir des fonctionnaires coloniaux en Guyane française au XVIII ${ }^{e}$ siècle, dir. G. MARTINIÈRE.

\section{INDEX}

Index géographique : La Rochelle 